\title{
Development of combined thymic carcinoma and thymoma in an extrathymic lesion during long follow-up for recurrent thymoma
}

\author{
YASUHIRO OHUE $^{1}$, SHUNICHIRO MATSUOKA ${ }^{1}$, HIROTAKA KUMEDA ${ }^{1}$, HIROYUKI AGATSUMA $^{1}$, \\ AKIRA HYOUGOTANI $^{1}$, MASAYUKI TOISHI ${ }^{1}$, TAKAYUKI SHIINA ${ }^{1}$, KAZUO YOSHIDA $^{1}$, \\ KUNIHIKO SHINGU ${ }^{2}$, TOSHIROU FUKUSHIMA ${ }^{3}$ and TOMONOBU KOIZUMI ${ }^{3}$ \\ ${ }^{1}$ Division of Thoracic Surgery, Department of Surgery; Departments of ${ }^{2}$ Laboratory Medicine \\ and ${ }^{3}$ Comprehensive Cancer Therapy, Shinshu University School of Medicine, Matsumoto, Nagano 390-8621, Japan
}

Received June 19, 2015; Accepted November 10, 2015

DOI: $10.3892 /$ mco. 2015.708

\begin{abstract}
The present study reported a rare case of combined thymic squamous cell carcinoma and thymoma exhibiting a mass on the left chest wall. The patient underwent thoracotomy for invasive thymoma 15 years previously, however, suffered a relapse in the left intrathoracic space. Radiotherapy, chemotherapy and partial resection, as secondary surgery for the intrathoracic mass, were performed. The histological findings in the resected specimens revealed type B3 thymoma. As the patient developed a left chest wall mass and pain in 2013, the mass was resected. The histological findings indicated two separate components composed of type B3 thymoma and squamous cell carcinoma. Immunohistological findings revealed that the thymoma cells were positive for CD5, while the thymic carcinoma cells were negative for CD5. Several reports have demonstrated the coexistence of thymic carcinoma and thymoma in the primary thymus, however, the development of a combined tumor in an extrathymic lesion is extremely rare. The present case had a long follow-up for recurrent thymoma. The present case indicated that the development and/or coexistence of malignant components in the thymoma must be taken into consideration for the treatment and/or management of patients with thymoma and that a pre-existence of CD5 expression in thymoma and the lost change may be associated with the process of malignant transformation.
\end{abstract}

\section{Introduction}

Thymoma is the most common neoplasm in the anterior mediastinum and $\sim 70 \%$ of the cases are well encapsulated,

Correspondence to: Professor Tomonobu Koizumi, Department of Comprehensive Cancer Therapy, Shinshu University School of Medicine, 3-1-1 Asahi, Matsumoto, Nagano 390-8621, Japan

E-mail: tomonobu@shinshu-u.ac.jp

Key words: thymic tumor, invasive thymoma, chemotherapy, tacrolimus, CD5 and therefore considered benign (1). However, thymoma has malignant potential and may invade into adjacent organs within the thorax or form distant metastasis. By contrast, thymic carcinoma differs from thymoma, not only morphologically, but also biologically (1-4). The majority of cases of thymic carcinoma appear to arise de novo, however, there have been rare reports of their occurrence and/or coexistence in thymomas (5-14). In addition, these combined thymic epithelial tumor types were located in the anterior mediastinum and diagnosed by first tumor resection.

The present study encountered a case of thymic carcinoma coexisting with thymoma developing on the chest wall in a patient who had received several chemotherapy regimens and thoracic radiotherapy for invasive thymoma associated with myasthenia gravis over a period of 15 years. The present study described the clinical course and a reviewed the relevant literature.

\section{Case report}

A 35-year-old woman, diagnosed with locally advanced thymoma [Masaoka et al classification, stage IVa (15)] underwent expanded thymectomy combined resection of the left brachiocephalic vein and left upper lung following four cycles of induction chemotherapy with cisplatin, doxorubicin, vincristine and cyclophosphamide in March 1999. The World Health Organization histological classification was type B3 (16). Subsequently, the patient received mediastinal radiotherapy (50 Gy). Relapse in the left pleural dissemination was observed in May 2003. As three cycles of carboplatin and paclitaxel chemotherapy failed to reduce the relapsed lesions, partial resection of the intrathoracic mass was performed as the second surgery. The patient developed dysphagia, ptosis and weakness in the neck in November 2010, and was diagnosed with anti-acetylcholine receptor antibody-positive myasthenia gravis. The patient was treated with prednisolone (10-15 mg/day) and tacrolimus (0.3 mg/day), which relieved and stabilized the symptoms of myasthenia gravis. However, tumors in the left intrathoracic space and chest wall grew in October 2013 (Fig. 1A). For the third-line setting, amrubicin administration was initiated and chest computed tomography (CT) following six cycles of amrubicin treatment demonstrated 
A

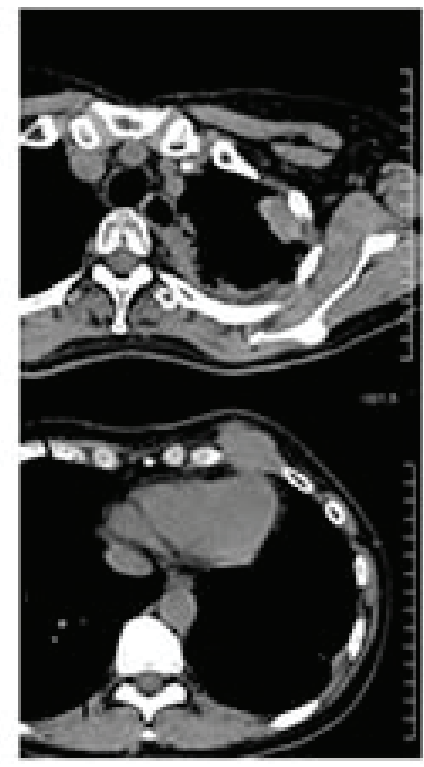

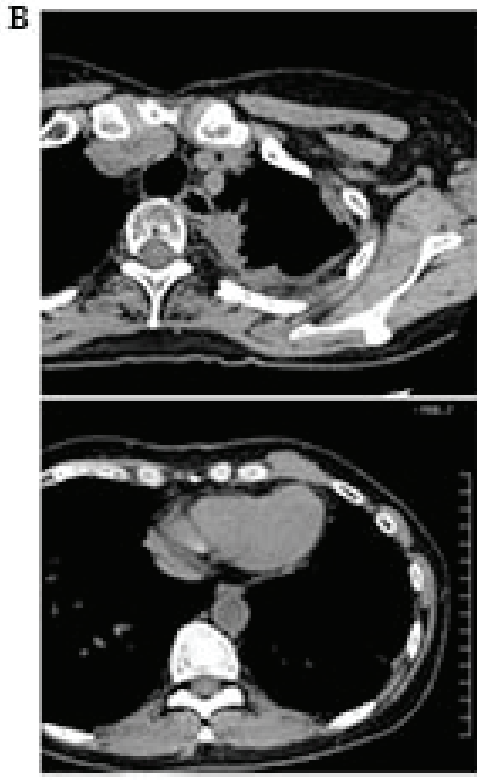

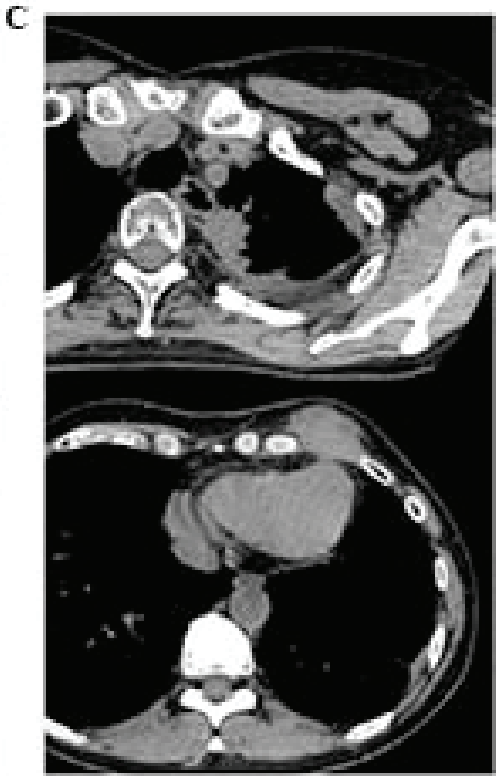

Figure 1. Chest computed tomography findings revealed left intrathoracic and chest wall masses (A) prior to and (B) following chemotherapy. (C) The chest wall mass increased in size 4 months following the cessation of chemotherapy.

a significant reduction in the size of the masses (Fig. 1B). Following 4 months of the last chemotherapy, the left intrathoracic tumor was stable, however the left anterior chest wall tumor grew again (Fig. 1C). The patient underwent chest wall tumor resection (Fig. 2) and the histopathological findings revealed two separate components of a mixed type with features of squamous cell carcinoma (Fig. 3A) and thymoma (Fig. 3B). Notably, immunohistological analysis indicated CD5 positivity in the thymoma area, however, negative in the thymic cancer area (Fig. 4). The post-operative course was uneventful and no evidence of residual tumor growth was detected 9 months following the final surgery.

\section{Discussion}

Combined thymic epithelial tumors are rare and characterized by at least two distinct areas each corresponding to one of the histological thymoma and thymic carcinoma types (5-14). Several previous reports indicated that the histological types between the thymic carcinoma and thymoma are not always correlated (5-14). However, Suster et al (6) summarized 22 cases of combined thymic epithelial tumor types and reported that the most common combination was typical B2 or B3, and squamous cell carcinoma. The pathological findings were consistent with the present case. The majority of cases of combined thymic tumor types reported to date were observed in the anterior mediastinum and diagnosed on first tumor resection. The present case developed a combined thymic epithelial tumor in the chest wall lesion at follow-up $>15$ years following the initial diagnosis. Therefore, the present case clearly indicated that thymic carcinoma can arise from a pre-existing thymoma.

The etiology of combined thymic epithelial tumor types remains enigmatic. Several previous studies indicated that patients with thymoma exhibited increased risk of developing second primary extrathymic malignancies $(17,18)$. In addition,

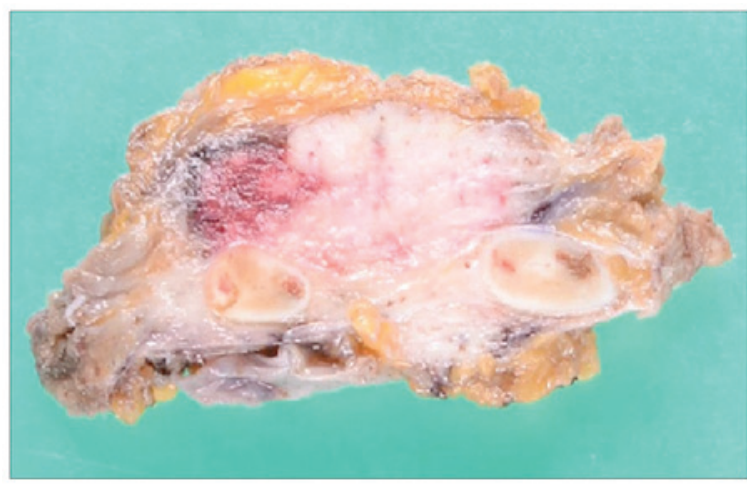

Figure 2. Macroscopic findings of resected tumor revealed major well-demarcated areas with light yellow and slight reddish areas within the mass.

myasthenia gravis is by far the most common paraneoplastic manifestation, and was also observed in the present case. It is unclear whether the presence of myasthenia gravis contributes to the development of combined thymic epithelial tumor types. However, the present study speculated that long-term administration of prednisolone and tacrolimus, and a history of several chemotherapies may have contributed to the malignant transformation in the present case. The patient received thoracic radiotherapy following the initial thoracotomy, however the location of the combined thymic epithelial tumor was slightly different compared with the previous radiation field. Therefore, it is likely that the previous thoracic radiotherapy caused no direct affect on the development of the malignant component in the present case.

Thymic carcinoma, however, not thymoma, is commonly immunoreactive for CD5 $(19,20)$. Notably, thymic squamous cell carcinoma is positive for CD5. However, serial immunohistological findings indicated the thymoma to be positive for CD5 in the present case, while the thymic carcinoma was negative for CD5. Therefore, CD5 immunoreactivity 

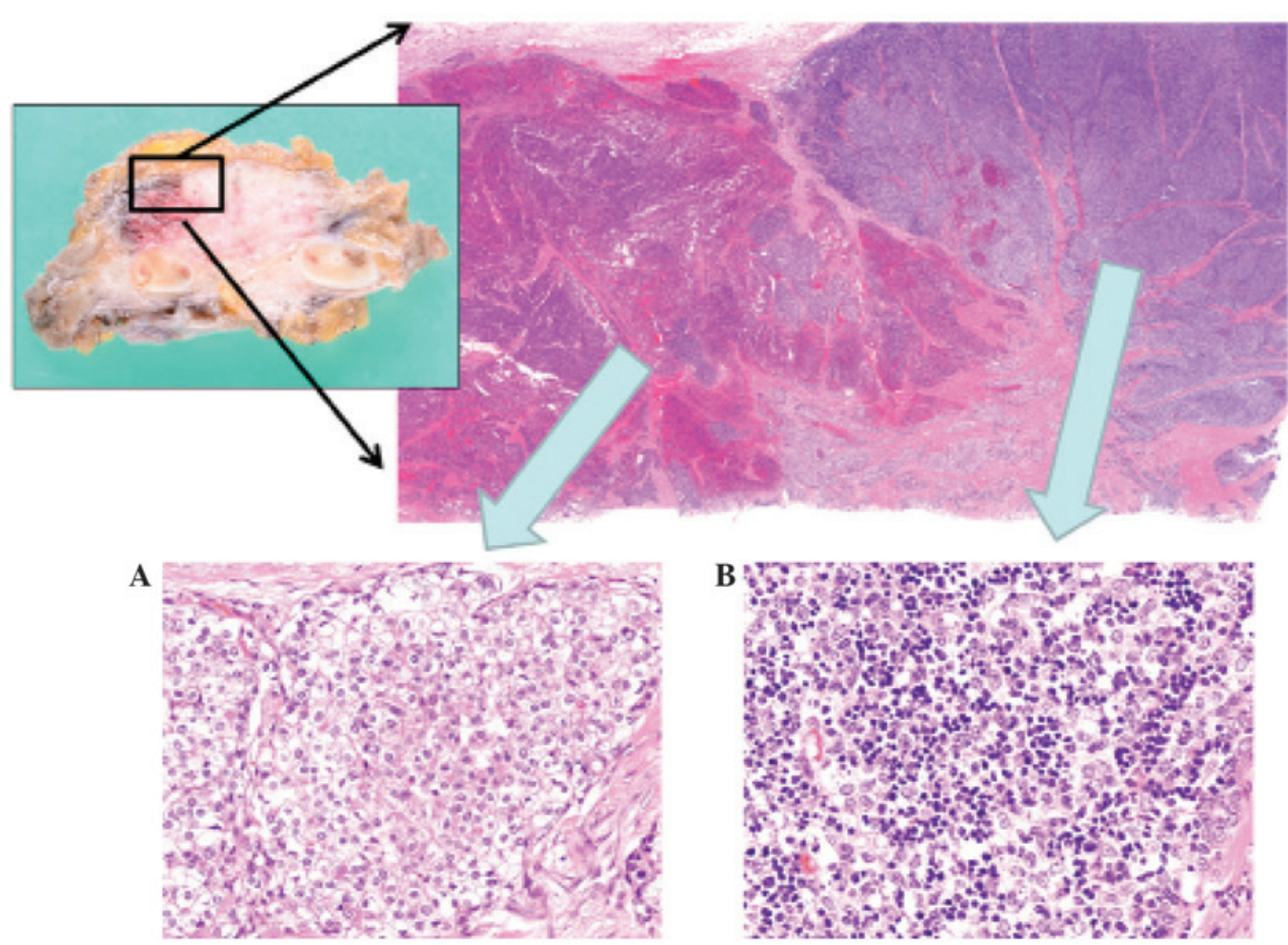

Figure 3. Micrograph of the left upper area of the resected tumor (square position). The area contained two separate components consisting of (A) squamous cell carcinoma and (B) type B2 thymoma.
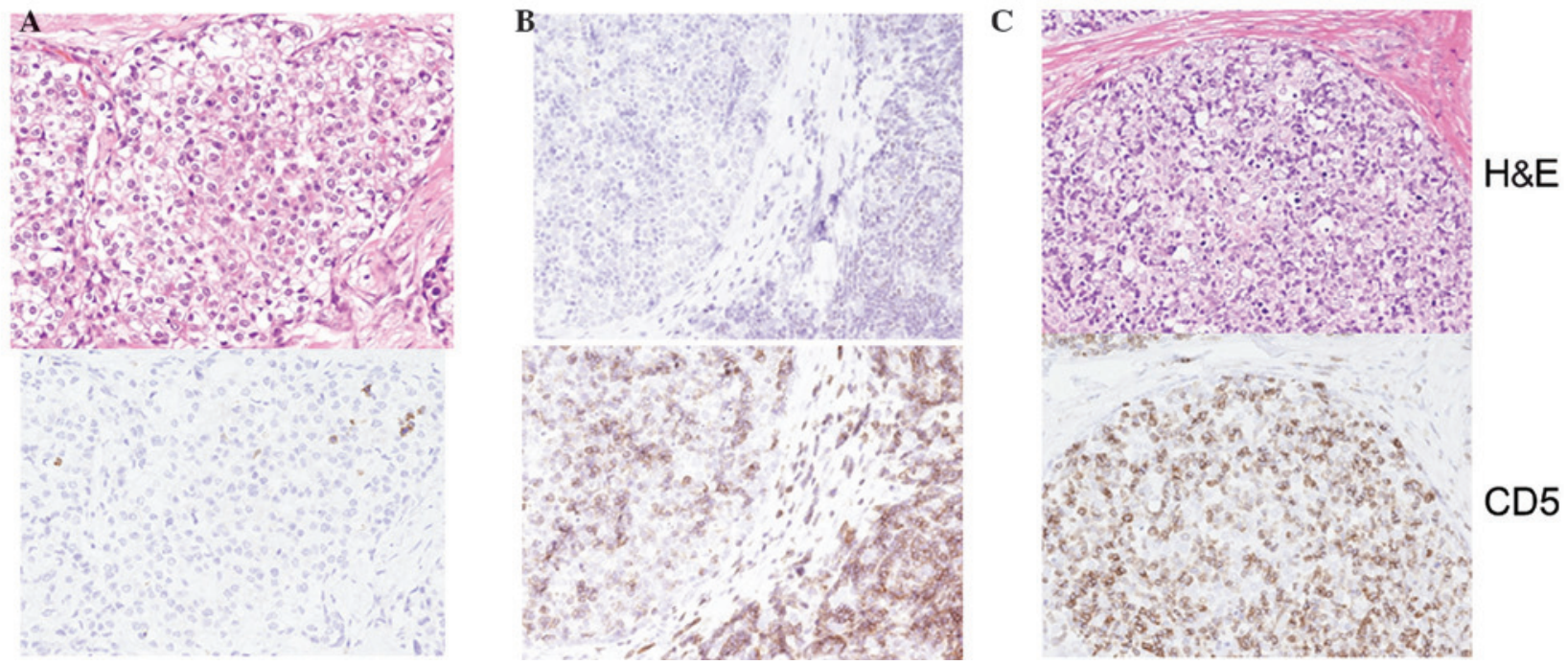

Figure 4. H\&E and immunohistochemical staining for CD5 in thymic carcinoma. (A) Tumor cell in squamous cell carcinoma were negative for CD5. The images show (B) the current combined tumor of thymoma and (C) previous thymoma resected by second salvage thoracotomy. The current and previous thymoma cells were positive for CD5. H\&E, hematoxylin and eosin; CD, cluster of differentiation.

was lost during malignant transformation in the present case. Suster et al (6) and Kuo et al (8) reported a similar case with a paradoxical change in the expression of CD5 in thymic epithelial tumors, respectively. The histological types (squamous cell carcinoma and type B3 thymoma) were also similar to those in the present study. These findings suggested that acquisition of CD5 immunoreactivity in thymoma cells may be an indicator of a biologically 'aggressive' phenotype. Further clinical experience and immunohistochemical studies are required to resolve the mechanism and/or interaction between malignant transformation to thymic carcinoma, and the change in CD5 expression.

As the immunoreactivity for CD5 was negative in the thymic carcinoma cells in the present case, there was a possibility of metastasis from other organs. However, two different thymic epithelial components coexisted in the resected chest wall tumor mass. Therefore, the present study hypothesized that the malignant tumor was not a metastatic tumor of other origins. Indeed, physical examination and ${ }^{18} \mathrm{~F}$-Fluorodeoxy glucose positron emission tomography findings revealed no 
abnormalities, with the exception of in the right thoracic space where recurrent thymoma was present, suggesting that no primary metastatic squamous cell carcinoma lesions existed.

In conclusion, the present study reported a rare case of combined thymic squamous cell carcinoma and thymoma in an extrathymic lesion, which developed over the course of long-term follow-up for recurrent thymoma associated with myasthenia gravis. The present case had a history of immunosuppressive agents and chemotherapy, however, suggested the possibility of malignant transformation in patients with thymoma associated with myasthenia gravis. The development and/or coexistence of malignant components in thymoma must be taken into consideration for the treatment and/or management of patients with thymoma.

\section{References}

1. Mikhail M, Mekhail Y and Mekhail T: Thymic neoplasms: A clinical update. Curr Oncol Rep 14: 350-358, 2012.

2. Hejna M, Haberl I and Raderer M: Nonsurgical management of malignant thymoma. Cancer 85: 1871-1884, 1999.

3. Kondo K and Monden Y: Therapy for thymic epithelial tumors: A clinical study of 1,320 patients from Japan. Ann Thorac Surg 76: 878-884, 2003

4. Suster S and Rosai J: Thymic carcinoma. A clinicopathologic study of 60 cases. Cancer 67: 1025-1032, 1991.

5. Muller-Hermelink HK, Strobel P,Zettl A and Marx A: Combined thymic epithelial tumors. In: WHO classification of tumors of the lung, pleura, thymus and heart. Travis WD, Brambilla E, Muller-Hermelink HK and Harris CC (eds). IARC Press, Lyon pp196-197, 2004.

6. Suster S and Moran CA: Primary thymic epithelial neoplasms showing combined features of thymoma and thymic carcinoma A clinicopathologic study of 22 cases. Am J Surg Pathol 20: 1469-1480, 1996.

7. Morinaga S, Sato Y, Shimosato Y, Sinkai T and Tsuchiya R: Multiple thymic squamous cell carcinomas associated with mixed type thymoma. Am J Surg Pathol 11: 982-988, 1987.

8. Kuo TT and Chan JK: Thymic carcinoma arising in thymoma is associated with alterations in immunohistochemical profile. Am J Surg Pathol 22: 1474-1481, 1998.
9. Wagner KB, Khan A, Mir R and Herman PG: Thymic carcinoma arising from long standing thymoma and presenting with pituitary metastases. Comput Med lmaging Graph 15: 411-414, 1991.

10. Suarez Vilela D, Salas Valien JS, Gonzalez Moran MA, lzquierdo Garcia F and Riera Velasco JR: Thymic carcinosarcoma associated with a spindle cell thymoma: An immunohistochemical study. Histopathology 21: 263-268, 1992.

11. Hartman CA, Roth C, Minck C and Niedobitek G: Thymic carcinoma. Report of five cases and review of the literature. J Cancer Res Clin Oncol 116: 69-82, 1990.

12. Shimosato Y, Kameya T, Nagaki K and Suemasu K: Squamous cell carcinoma of the thymus: An analysis of eight cases. Am J Surg Pathol 1: 109-121, 1977.

13. Wu SG, Li Y, Li B, Tian XY and Li Z: Unusual combined thymic mucoepidermoid carcinoma and thymoma: A case report and review of literature. Diagn Pathol 9: 8, 2014.

14. Kuo T, Chang JP, Lin FJ, Wu WC and Chang $\mathrm{CH}$ : Thymic carcinomas: Histopathological varieties and immunohistochemical study. Am J Surg Pathol 14: 24-34, 1990.

15. Masaoka A, Monden Y, Nakahara K and Tanioka T. Follow-up study of thymomas with special reference to their clinical stages. Cancer 48: 2485-2492, 1981.

16. Travis WD, Brambilla E, Muller-Hermelink HK and Harris CC (eds): Pathology and genetics of tumours of the lung, pleura, thymus and heart. In: WHO Classification of Tumors of the Lung, Pleura, Thymus and Heart. IARC press, Lyon, 2004.

17. Filosso PL, Galassi C, Ruffini E, Margaritora S, Bertolaccini L, Casadio C, Anile M and Venuta F: Thymoma and the increased risk of developing extrathymic malignancies: A multicentre study. Eur J Cardiothorac Surg 44: 219-224, 2013.

18. Gadalla SM, Rajan A, Pfeiffer R, Kristinsson SY, Björkholm M, Landgren $\mathrm{O}$ and Giaccone G: A population-based assessment of mortality and morbidity patterns among patients with thymoma. Int J Cancer 128: 2688-2694, 2011.

19. Dorfman DM, Shahsafaei A and Chan JK: Thymic carcinomas, but not thymomas and carcinomas of other sites, show CD5 immunoreactivity. Am J Surg Pathol 21: 936-940, 1997.

20. Hishima T, Fukayama M, Fujisawa M, Hayashi Y, Arai K, Funata N and Koike M: CD5 expression in thymic carcinoma. Am J Pathol 145: 268-275, 1994. 